\title{
Tourisme médical et santé reproductive : l'exemple de la gestation pour autrui en inde
}

\section{Virginie Chasles et Marion Girer}

\section{CpenEdition}

Journals

Édition électronique

URL : http://journals.openedition.org/rfst/514

DOI : $10.4000 /$ rfst.514

ISSN : 2492-3672

Éditeur

Espaces et SOciétés (UMR 6590)

Référence électronique

Virginie Chasles et Marion Girer, «Tourisme médical et santé reproductive : l'exemple de la gestation pour autrui en inde », Revue francophone sur la santé et les territoires [En ligne], Tourisme, Mobilités et Santé, mis en ligne le 25 octobre 2016, consulté le 06 avril 2021. URL : http://journals.openedition.org/ rfst/514 ; DOI : https://doi.org/10.4000/rfst.514

Ce document a été généré automatiquement le 6 avril 2021.

\section{(i) (2)}

La Revue francophone sur la santé et les territoires est mise à disposition selon les termes de la Licence Creative Commons Attribution - Pas d'Utilisation Commerciale - Partage dans les Mêmes Conditions 4.0 International. 


\title{
Tourisme médical et santé reproductive : l'exemple de la gestation pour autrui en inde
}

\author{
Virginie Chasles et Marion Girer
}

\section{Introduction}

1 Nos sociétés contemporaines sont confrontées à de multiples questions nouvelles, issues notamment du développement des moyens de transport et de communication et de l'accroissement des connaissances et techniques médicales. Si ces évolutions constituent une source indéniable d'amélioration de la qualité de vie des populations, au moins dans les pays qui peuvent y avoir accès, elles génèrent cependant des situations et des risques inédits auxquels le droit peine à répondre, tant les enjeux éthiques et sociétaux sont fondamentaux. Le tourisme médical est une illustration de ces problématiques émergentes en matière de santé, au niveau international. Cette expression se réfère au déplacement d'individus, malades ou bien portants, allant dans un pays autre que leur pays de résidence, dans le but de bénéficier d'un acte médical non disponible ou difficilement accessible dans leur propre pays, soit pour des raisons de législation, soit pour des raisons relatives à l'offre de soins (compétences, coût).

2 Si l'expression de tourisme médical englobe une diversité de prestations médicales, allant de la simple chirurgie plastique à des soins beaucoup plus lourds (chirurgie cardiaque, greffe d'organes...), une place à part doit être réservée en son sein au tourisme procréatif, défini comme le fait de se rendre à l'étranger pour bénéficier de techniques médicales permettant de donner naissance à un enfant. A travers l'exemple de l'Inde, l'un des pôles mondiaux du tourisme médical contemporain, cet article vise à mettre en lumière une dimension encore peu documentée du tourisme médical. A partir d'une approche multiscalaire, il s'agira de montrer dans quelle mesure la géographie du tourisme procréatif obéit à des logiques différentes de celles du tourisme médical. En effet, alors que dans ce dernier cas, l'attractivité des territoires est liée 
avant tout au coût et à la qualité des prestations médicales offertes, dans le cas du tourisme procréatif, c'est la possibilité concrète d'avoir recours à certaines pratiques qui oriente les flux. Fondé sur l'exemple de la gestation pour autrui, cet article se propose de dresser un bilan de cette pratique en Inde et d'en évaluer l'impact sur les territoires concernés, en termes plus particulièrement de développement d'infrastructures de santé et d'aménités touristiques.

\section{L'Inde, une destination touristique secondaire mais un pôle du tourisme médical}

3 En 2014, l'Union indienne, 41 ème destination touristique mondiale, a enregistré l'arrivée de 8 millions de touristes étrangers (ce qui la situe au niveau du Portugal ou de la Belgique). A titre comparatif, la même année, la Chine, 5 ème pays récepteur, a reçu 37 millions de touristes étrangers (et la France, 1ère destination touristique au monde, en a reçu environ 85 millions). Avec 20,2 milliards de dollars de recettes, l'Inde ne représente que $1,58 \%$ des recettes touristiques mondiales et n'accueille que $0,68 \%$ des touristes étrangers dans le monde (Indian Tourism Statistics at a Glance, 2014). L'Inde fait donc figure de destination de second rang à l'échelle mondiale, même si elle compte parmi les pays ayant enregistré les plus fortes hausses de fréquentation ces dernières années. En effet, entre 2013 et 2014, le nombre de touristes internationaux en Inde a augmenté de 10,2\%. Cependant, il s'agit d'un secteur empreint d'incertitudes liées aux fluctuations de la conjoncture économique, aux aléas naturels (tremblement de terre au Gujarat en 2001, tsunami sur les côtes de l'Inde du Sud en 2004, inondations au Tamil Nadu en 2015...) et sociopolitiques (affrontements intercommunautaires à Ahmedabad en 2002, attentats meurtriers dans cette même ville en 2008, attentats à Mumbai et Jaipur en 2008, guérilla naxalite dans l'est du pays). A cette incertitude de la demande, s'ajoute aussi la concurrence entre les destinations d'Asie du Sud-Est (Bautès \& al., 2015). Depuis quelques années, pour attirer les touristes étrangers, le pays a engagé une campagne de promotion touristique. En s'appuyant sur le slogan «Incredible India », l'objectif est de valoriser certains territoires mais aussi certains aspects de la culture indienne (fêtes hindoues, circuit Ramayana ${ }^{1}$, circuit bouddhiste ${ }^{2}$...) à travers des offres innovantes, originales et lucratives. C'est dans ce cadre qu'est encouragé le développement du tourisme de bien-être, basé notamment sur la pratique du yoga et le recours à l'ayurvéda, et du tourisme médical.

4 Concernant ce second aspect, l'Inde constitue d'ores et déjà un pôle majeur du tourisme médical (Chasles, 2011). De manière générale, depuis la fin des années 1990, les patients internationaux se rendent principalement vers les pays émergents. Plusieurs raisons peuvent être identifiées. Dans les pays de départ, on peut évoquer les délais d'attente importants pour certains soins, l'absence de soins de qualité ou bien encore les contraintes de législation (interdiction ou restriction de certaines pratiques). Dans les pays d'accueil, le faible coût des soins est souvent le facteur le plus attractif. Avant 1997, les Etats-Unis et l'Europe étaient les centres du tourisme médical, les patients aisés des pays émergents et en développement venant s'y faire soigner. Mais la crise économique qui toucha l'Asie du Sud entre 1997 et 2001 changea profondément ces logiques spatiales. En effet, d'une part les patients asiatiques n'ont plus eu les capacités financières de se rendre à l'étranger pour se faire soigner et, d'autre part, cette crise financière a rappelé la nécessité de diversification de l'économie locale (Heng Leng, 2007). C'est ainsi que certains pays asiatiques, comme la Thaïlande, la 
Malaisie ou encore l'Inde, ont décidé de développer davantage ce secteur pour retenir leurs propres patients mais aussi pour attirer ceux des autres pays. En outre, cette tendance s'est trouvée renforcée par les attaques terroristes de 2001 aux Etats-Unis, qui découragèrent les patients des pays arabes de se rendre sur le territoire américain pour se faire soigner.

5 Apparu au cours des années 1990, à la suite de la libéralisation et de la mondialisation de l'économie indienne, le tourisme médical est un secteur en pleine expansion en Inde. A l'échelle de l'Asie, le pays fait partie des trois principales destinations des touristes médicaux avec la Thaïlande et la Malaisie (Qadeer \& Reddy, 2010). Depuis le début des années 2000, l'Etat encourage ce secteur en proposant des allègements de taxes pour les professionnels, en diminuant les taxes sur l'importation d'équipement médical ou bien encore en facilitant l'obtention de visas pour ces touristes (Anand \& Gupta, 2008). Concernant ce dernier aspect, depuis 2005, le gouvernement indien a mis en place une politique de visa spécifique ${ }^{3}$. D'une durée d'un an, les visas médicaux sont renouvelables par simple lettre d'un médecin indien, pour les patients ainsi que pour les accompagnants (Baujard, 2012). Depuis 2015, ces visas médicaux sont gratuits pour plus de 180 nationalités, ce qui va fort probablement constituer un nouvel élan pour ce secteur. Au total, le nombre de touristes médicaux en Inde serait passé de 150000 personnes en 2005 à 450000 en 2008 (Chinai \& Goswami, 2008). Les facteurs attractifs sont le faible coût des soins, la qualité des hôpitaux et le haut niveau de compétence des médecins, un certain nombre d'entre eux ayant été formés dans les pays occidentaux. Ce secteur, principalement porté par de grands groupes hospitaliers privés (Bochaton et Lefebvre, 2008), aurait enregistré un chiffre d'affaires de plus de 2 milliards de dollars en 2012 (Solomon, 2011). Pour s'assurer la confiance des patients, ces hôpitaux sollicitent une accréditation auprès de la Joint Commission International. Cette autorité médicale internationale évalue plus de 1030 critères portant notamment sur la sécurité du patient et la qualité des soins. A ce jour, 21 hôpitaux indiens ont obtenu cette accréditation. Les soins les plus convoités relèvent de différents domaines de la chirurgie (interventions cardiaques, greffes de moelle osseuse, prothèses de la hanche, chirurgie de l'oeil...) et aussi des soins de bien-être inspirés de la médecine traditionnelle ayurvéda.

6 Ces patients internationaux sont originaires avant tout d'Asie, et plus particulièrement du Bangladesh et du Sri Lanka. Ils sont également nombreux en provenance de Malaisie et du Pakistan. S'ajoutent ensuite les patients originaires des pays développés (EtatsUnis, Royaume-Uni). Parmi ces derniers, les Indiens de la diaspora sont nombreux. A ce sujet, de nombreuses études sur la santé des immigrés ont montré que le retour au pays d'origine pour se faire soigner était relativement fréquent. Les raisons sont multiples. Certaines sont de l'ordre des croyances médicales, des préférences culturelles, des barrières linguistiques ou encore des difficultés d'intégration dans le pays d'accueil (Gany \& al., 2006). Il est alors considéré que le processus de guérison n'est pas simplement influencé par le degré de qualification médicale mais aussi par la proximité culturelle et émotionnelle vis-à-vis du lieu de prise en charge. Ceci fait référence à la notion de "paysage thérapeutique" développée par W. Gesler, qui sous-tend l'idée que certains espaces, comme le pays d'origine ou le lieu de résidence, de par leur charge symbolique et sociale, peuvent être source de bien-être (Gesler, 1992).

7 Cette expression de tourisme médical recoupe également ce qu'on appelle le tourisme procréatif. Depuis quelques années, l'Inde est une destination de plus en plus prisée 
dans le domaine de la procréation médicalement assistée, et plus particulièrement dans celui de la gestation pour autrui (Pande, 2011). Si ce secteur est englobé dans celui plus large du tourisme médical, il n'en demeure pas moins qu'il s'en distingue nettement, de par les logiques qui concourent au choix de l'Inde comme lieu de prise en charge.

\section{Le tourisme "procréatif », une expression impropre pour une pratique grandissante}

tourisme procréatif, défini comme le fait de se rendre à l'étranger pour bénéficier de techniques médicales permettant de donner naissance à un enfant, est parfois qualifié de " délocalisation procréative » (Mouly, 2014), d'« usage transnational de l'assistance médicale à la procréation (Brunet, 2012), voire d'« externalisation de la fabrique des enfants » (Hauser, 2014). Le tourisme procréatif présente deux particularités. Tout d'abord, il a, dans la quasi-totalité des cas, un caractère illicite, dans la mesure où son but principal est de contourner une interdiction posée par la loi du pays dont on possède la nationalité ou dans lequel on vit : il vise à obtenir ce que le droit national prohibe. Parfois, des considérations financières entrent également en jeu, lorsqu'il s'agit d'obtenir une prestation ou un service à un prix plus avantageux hors de ses frontières nationales, mais le plus souvent c'est la prohibition qui guide à titre principal le choix de se déplacer à l'étranger. Ensuite, une autre spécificité du tourisme procréatif est le fait d'utiliser les techniques de l'assistance médicale à la procréation (AMP) pour aboutir à la naissance d'un enfant: il ne s'agit pas vraiment pour les personnes de bénéficier de prestations de santé pour elles-mêmes, mais de recourir à une aide médicale et technique pour fonder une famille. Entrent ainsi dans le cadre du tourisme procréatif l'insémination artificielle d'une femme seule; l'insémination artificielle d'une femme, seule ou en couple, ayant dépassé la limite d'âge prévue par la législation de son pays pour profiter d'une telle technique ; l'insémination artificielle d'une femme dont le mari est décédé et qui demande une insémination post-mortem ; ou encore le recours à une gestation pour autrui.

Dans la mesure où le tourisme procréatif se développe afin d'échapper aux règles de droit en vigueur dans un pays, il conduit nécessairement à des déplacements à l'étranger et à un flux de demandes vers des pays plus permissifs. L'interdiction, la tolérance ou la permission juridique sont donc autant de critères qui permettent de proposer une explication, non pas unique, mais opérante, à ce déplacement d'un territoire de santé à l'autre dans un but de procréation. Seul l'exemple de la gestion pour autrui sera examiné dans cet article, car il est emblématique des difficultés fondamentales soulevées par cette forme de tourisme procréatif, en termes juridiques, éthiques et sociétaux : il se situe entre l'enclume de la loi et le marteau des pratiques (Brunet, 2010).

Dans le langage courant, l'expression de "mères porteuses" est le plus souvent utilisée. Le langage juridique préfère la notion générale de maternité de substitution ou de maternité pour autrui, définie comme le fait de porter un enfant en vue de le remettre soit à un couple, hétérosexuel ou homosexuel, soit à une personne seule. Au sein de la maternité de substitution, il faut distinguer d'une part la procréation pour autrui, lorsque la femme qui porte l'enfant est aussi la génitrice, c'est-à-dire la mère génétique de l'enfant, et d'autre part la gestation pour autrui (Fabre-Magnan, 2013 ; Schamps \& Sosson (dir.), 2013), hypothèse dans laquelle l'enfant est conçu à partir de 
l'ovocyte de la mère d'intention ou d'une tierce personne donneuse : la gestatrice n'est alors pas la mère génétique de l'enfant, elle ne fait que le "porter " pour autrui. Dans tous les cas, il est prévu que l'enfant soit remis à sa naissance au(x) parent(s) d'intention, désigné(s) par les termes de couple d'intention, de couple d'accueil ou encore de couple commanditaire.

11 Si la maternité de substitution est un phénomène aujourd'hui largement médiatisé, il s'agit en réalité d'une pratique séculaire, restée longtemps discrète. Elle est ainsi mentionnée dans la Bible: les épouses d'Abram et de Jacob, ne pouvant enfanter, avaient demandé à leurs servantes de porter des enfants à leur place. La maternité de substitution s'est ensuite poursuivie dans le temps, tolérée sans être reconnue, souvent dans le secret des familles pour venir en aide à une femme stérile. La discrétion du cercle familial a cédé la place à une médicalisation possible et à un regard de la société sur cette méthode de conception d'un enfant, faisant passer la procréation « du secret des alcôves à la médicalisation » (Manaï, 2013). Ainsi, la gestation pour autrui connaît depuis quelques années un essor considérable, et ceci pour plusieurs raisons, parmi lesquelles l'augmentation de la stérilité, la fermeture des voies de l'adoption et aussi le désir de partager un lien génétique avec l'enfant à venir. Ce dernier aspect est particulièrement important dans le contexte culturel de l'Inde. En effet, à la différence de l'adoption, la gestation pour autrui permet de transmettre le patrimoine génétique des parents à l'enfant, et par là même son patrimoine culturel, sa religion et sa caste. Cet aspect explique que les Indiens eux-mêmes, du territoire national et de la diaspora, soient toujours plus nombreux à recourir à cette pratique.

12 L'évolution des techniques médicales dans le domaine de la procréation a fait naître de nouvelles questions éthiques, morales et juridiques. Pour le juriste, le principal écueil est celui de la délimitation des frontières : jusqu'où peut-on aller pour permettre à un couple, ou à un individu seul, de réaliser son désir d'enfant grâce à une aide médicalisée ? L'assistance médicale à la procréation interroge les conceptions de la parenté et de la parentalité dans notre société contemporaine (Fulchiron, 2013). Jusqu'à présent, le législateur français a fait le choix d'une interdiction de la gestation pour autrui, mais les débats font rage autour du maintien ou de l'abandon de cette prohibition. Si la France interdit expressément le recours à la maternité de substitution, d'autres pays ont adopté un régime plus permissif, basé soit sur l'absence de réglementation particulière, soit sur l'autorisation formelle.

Dans le cadre de cette étude, il ne s'agit pas de dresser un panorama exhaustif de la législation de chaque pays relative à la gestation pour autrui, mais plus modestement de montrer à travers quelques exemples qu'il existe à la fois une diversité des législations nationales et une absence de règle contraignante au niveau international (même si d'importantes discussions ont actuellement lieu dans le cadre du Conseil de l'Europe), ce qui laisse aux Etats une marge de manœuvre importante. Ce contexte général participe au développement de la maternité de substitution dans certains pays, notamment en Inde. Ainsi, plusieurs pays ont fait le choix, comme la France, d'interdire expressément la gestation pour autrui dans leurs corpus juridique. Il s'agit notamment de l'Allemagne, de l'Espagne, de l'Italie, de l'Autriche, de la Suède ou encore du Québec. Le Portugal faisait traditionnellement partie de cette catégorie, mais une loi a été votée en mai 2016 pour autoriser le recours à la gestation pour autrui, à titre exceptionnel et gratuit, pour les femmes qui ne peuvent pas porter d'enfant pour des raisons médicales (absence d'utérus, lésion ou maladie de cet organe qui empêche de façon absolue et 
définitive la grossesse, ou quand la situation clinique le justifie). Il n'est cependant pas certain que le texte puisse s'appliquer à l'avenir, dans la mesure où le Président de la République a opposé son véto à ce texte quelques semaines après son vote.

Certains pays ont à l'inverse expressément autorisé le recours à la gestation pour autrui, sous conditions plus ou moins strictes : Royaume-Uni, Australie, Afrique du Sud, Argentine, Brésil, Géorgie, plusieurs états des Etats-Unis, Grèce, Irlande, Ukraine, Russie... La législation permet alors d'instaurer un lien de filiation avec le ou les parents d'intention, soit légal, soit contractuel. Les enjeux principaux concernent l'indemnisation ou la rémunération de la gestatrice, la force juridique accordée à la volonté de chacun des acteurs quant au déroulement de la grossesse (suivi médical, observance des traitements, interruption de grossesse...) et l'établissement de l'acte de naissance.

Enfin, d'autres pays se situent dans une situation intermédiaire, du fait de l'absence d'interdiction ou d'autorisation expresse, ce qui conduit à une tolérance de la pratique de la gestation pour autrui, sans réel encadrement.

Il s'agit par exemple de la Belgique, du Danemark, de la Hongrie, de la Pologne, des Pays-Bas ou bien encore de l'Inde. Ce contexte législatif «flottant », associé aux faibles tarifs pratiqués, fait que l'Inde est rapidement devenue une destination privilégiée pour le tourisme procréatif, et plus particulièrement pour le recours à la gestation pour autrui. Ainsi, si l'expression de "tourisme procréatif» est impropre, puisque la récréation n'est pas l'objectif fondamental de ce déplacement (Knafou \& Stock, 2003), il n'en demeure pas moins un phénomène grandissant. Le tourisme procréatif en Inde, un outil de développement touristique des territoires?

En Inde, la gestation pour autrui est tolérée depuis 2002. A ce jour, il n'y a toujours pas de loi encadrant cette pratique mais simplement des recommandations émises par l'Indian Council of Medical Research et examinées par le Gouvernement dans le cadre de projets de lois successifs mais non ratifiés. Il existe toutefois, depuis 2012, une loi qui interdit le recours à la gestation pour autrui pour les célibataires et les couples homosexuels. Au cours des dernières années, l'Inde est devenue peu à peu une destination privilégiée pour le recours à la gestation pour autrui. Elle est parfois qualifiée de « low cost » de la gestation pour autrui, puisqu'elle y coûte entre 25000 et 35000 dollars, contre 70000 à 100000 dollars aux Etats-Unis (Rozée Gomez \& Unisa, 2014). Les raisons du développement de ce secteur sont en réalité multiples, parmi lesquelles on peut citer l'absence de contraintes légales (Rozée \& Tain, 2010), la médiatisation et la croissance de l'offre dans ce domaine, les compétences reconnues dans le domaine de la santé reproductive et aussi la proximité linguistique permise par l'usage de l'anglais (Rozée Gomez \& Unisa, 2014). Cependant, il faut bien noter que la croissance de cette pratique en Inde est avant tout une illustration des mobilités produites par l'évolution du contexte légal qui encadre la procréation médicalement assistée de manière générale, et la gestation pour autrui en particulier. Ainsi, la cartographie des "patients » et leurs logiques de mobilité s'ajustent continuellement aux évolutions spatiales de la loi. Dans ce cas de figure, comme c'est d'ailleurs le cas pour d'autres types de soins (chirurgie bariatrique, interruption volontaire de grossesse, traitements à partir de cellules souches ...), on voit bien que ce qui régit d'abord les itinéraires des usagers de la gestation pour autrui, ce n'est pas tant le prix de ce service, ni le lieu de prise en charge, ni la qualité de la prestation médicale, que la possibilité d'y avoir recours. Curtin \& Inhorn (2011), ont d'ailleurs bien montré que la 
motivation première dans le recours aux soins reproductifs transfrontaliers est l'interdiction (légale ou religieuse) de certaines pratiques dans le pays de résidence.

Le choix entre différentes possibilités spatiales est ensuite dicté par des considérations financières. De ce point de vue, les flux qui animent le tourisme médical illustrent les mobilités produites par les inégalités de développement qui perdurent entre les territoires et les individus. A ce titre, le profil des « mères porteuses » en Inde est tout à fait significatif de la paupérisation des femmes dans certaines parties du monde et de la commercialisation qui est faite de cette pauvreté. Ainsi, les femmes qui s'engagent dans la gestation pour autrui sont majoritairement sans éducation et issues de classes sociales défavorisées, n'ont pas d'activités professionnelles stables ou suffisamment rémunératrices, et expriment de forts besoins financiers (pour l'achat d'un logement, pour le financement des études des enfants, pour le remboursement de dettes, pour compenser l'absence de travail de l'époux...). Devenir « mère porteuse » apparaît alors comme une stratégie de survie (Pande, 2010).

L'absence de données exhaustives et fiables ne permet pas d'évaluer précisément l'importance de cette pratique en Inde. Cependant, les quelques études menées dans ce domaine s'accordent sur la rapidité de sa croissance (Palattiyil, 2010). Si le tourisme procréatif attire les occidentaux, ces touristes sont également nombreux en provenance du Sri Lanka, du Pakistan, du Bangladesh, de la Thaillande ou bien encore de Singapour. Au total, certaines sources évaluent à près de 12000 le nombre d'étrangers qui viendraient chaque année en Inde pour recourir à cette pratique. Parmi eux, les membres de la diaspora indienne sont particulièrement nombreux. Seconde diaspora au monde avec environ 28 millions d'individus, ses membres préfèrent avoir recours à la gestation pour autrui en Inde, et ceci avant tout pour des raisons affectives et culturelles, alors même que certains résident dans des pays dans lesquels la gestation pour autrui est autorisée (Royaume-Uni, certains états des Etats-Unis). Par ailleurs, il faut noter que les couples étrangers ne sont pas les seuls à recourir à la gestation pour autrui. En effet, il y a une forte demande locale pour des raisons à la fois médicale et sociale. D'un point de vue médical, la stérilité concerne en Inde plus de 8 millions de personnes. Or, une fois mariée, la jeune femme a le devoir de devenir mère. La maternité lui confère une légitimité que rien d'autre ne saurait lui apporter: prospérité, éducation ne compensent pas l'absence d'enfant (Chasles, 2008). Ainsi, chaque grossesse délivre-t-elle de l'insécurité et de la honte qui entoure l'infertilité, la responsabilité de la stérilité étant le plus souvent attribuée à la femme même si elle touche davantage les hommes. Ainsi, même si recourir à la gestation pour autrui est dévalorisé socialement, cette stigmatisation est moindre comparativement au fait de ne pas avoir d'enfant. Ce devoir d'être mère a donc peu à peu permis le développement du secteur de la procréation médicalement assistée. Cependant, comme l'expliquent Rozée \& Unisa (2014), la disponibilité et l'accessibilité de ces techniques médicales créent d'importantes inégalités puisqu'elles sont proposées essentiellement par le secteur privé, qui pratique des prix prohibitifs pour une grande partie de la population. Localement, l'offre existante dans ce domaine bénéficie donc essentiellement aux catégories sociales les plus aisées (Rozée Gomez \& Unisa, 2014).

21 Si l'offre locale reste très peu accessible à la population, elle a toutefois permis de créer les conditions nécessaires à l'accueil de touristes médicaux étrangers. Ainsi, l'augmentation du recours à la gestation pour autrui en Inde est liée, comme vu précédemment, à la souplesse du cadre législatif et aux faibles prix pratiqués, mais 
aussi à l'existence de structures médicales pratiquant la gestation pour autrui, dont certaines de qualité répondant aux standards internationaux. Au total, selon la Commission Nationale pour les Femmes, il y aurait en Inde environ 3000 cliniques pratiquant la gestation pour autrui. Concentrées dans les grands centres urbains, elles privilégient ceux dotés de services et d'aménités nécessaires à l'accueil de touristes médicaux (hébergements, consulats...).

22 A l'échelle nationale, si ces cliniques sont présentes dans l'ensemble du territoire indien, c'est l'état du Gujarat qui bénéficie de la plus grande visibilité dans ce domaine, ce qui lui vaut parfois le qualificatif de "berceau du monde». Certaines de ses cliniques, telle celle de la fameuse Docteur Patel à Anand, ont développé des stratégies de communication, aux niveaux national et international, pour accroître leur attractivité auprès des "futurs parents ", mais aussi auprès des «mères porteuses ". Dans le premier cas, les discours commerciaux vantent la qualité des prestations médicales et la compétitivité des prix pratiqués. Dans le second cas, pour s'assurer un recrutement suffisant, ces cliniques mettent en lumière, abusivement, les opportunités financières qu'offre la gestation pour autrui. Concernant ce dernier aspect, précisons simplement que les «mères porteuses » ne reçoivent qu'une faible partie de la somme acquittée par les usagers de la gestation pour autrui, la plus grande partie étant accaparée par les cliniques elles-mêmes. Il s'agit là de l'un des nombreux problèmes éthiques soulevés par cette pratique en Inde.

23 Dans tous les cas, le Gujarat apparaît comme un haut-lieu du tourisme procréatif et compte déjà plusieurs cliniques mondialement connues, localisées plus particulièrement dans les villes d'Anand, de Surat et de Jamnagar. D'un point de vue économique, cet état est original de par l'importance des réformes économiques qui y ont été menées au cours des vingt dernières années. Dirigé jusqu'en 2014 par l'actuel Premier Ministre, Narendra Modi, cet état affiche un taux de croissance économique supérieur à la moyenne nationale ( $12 \%$ en 2011 contre un peu moins de $9 \%$ à l'échelle nationale) du fait plus particulièrement de l'importance de son tissu industriel. Si cet état est attractif du point de vue des investisseurs, il l'est par contre beaucoup moins pour les touristes. En effet, en 2014, cet état n'a accueilli que 2,4\% des touristes domestiques (soit environ 31 millions de personnes) et 1,04\% des touristes étrangers (soit environ 236000 personnes) (Ministère du tourisme du Gujarat, 2015). Par ailleurs, en 2014, dans son rapport annuel, le Ministère du tourisme a révélé que $53 \%$ des visiteurs viennent pour les affaires, $35 \%$ pour des raisons religieuses et $8 \%$ seulement pour les loisirs. Cette faible attractivité touristique n'est pas liée au manque de potentiels dans ce domaine. Bien au contraire, l'état dispose d'un patrimoine historique riche. Mais celui-ci reste mal exploité par les autorités municipales et l'industrie touristique (Milbert, 2015). Dans le même ordre d'idée, le Gujarat ne fait pas partie des destinations privilégiées par les touristes médicaux, ces derniers préférant les villes de Chennai ${ }^{4}$, Delhi, Mumbai ou bien encore Bangalore. En revanche, il est à noter que le Ministère $\mathrm{du}$ Tourisme du Gujarat souhaite clairement développer le tourisme médical et le tourisme de bien-être, considérés comme des niches innovantes pour le développement touristique. Le Ministère a un rôle de promotion, de soutien financier et aussi d'accompagnement des acteurs de ces deux domaines pour l'obtention d'accréditations nécessaires à leur visibilité et à leur légitimité (Ministère du tourisme du Gujarat, 2015). Pour le développement de son secteur touristique, l'état du Gujarat peut également compter sur sa puissante diaspora. En effet, au-delà du poids économique de la diaspora gujaratie, il est à noter que cette communauté contrôle près 
de $40 \%$ de l'offre hôtelière aux Etats-Unis. Elle peut donc constituer un acteur important du développement du tourisme dans son Etat d'origine.

A travers cet exemple du Gujarat, on voit bien dans quelle mesure le tourisme médical peut être mobilisé pour participer à la mise en tourisme de certains territoires. Les acteurs impliqués dans ce secteur ont pleinement conscience de la manne financière qu'il peut représenter. Mais celle-ci repose en grande partie sur la capacité des territoires à attirer les touristes médicaux. Ce potentiel d'attractivité est conditionné par la qualité de l'offre de soins et la nature des soins proposés, par la visibilité et ainsi la légitimité des territoires porteurs de ce secteur et aussi par la qualité des aménités touristiques (mise en valeur du patrimoine local, offre d'hébergements répondant aux standards internationaux, accessibilité des lieux...). Le développement du tourisme médical est donc en partie conditionné par celui du secteur du tourisme. Dans le même temps, le tourisme médical est un élément dynamisant le tourisme de bien-être, les convalescents et leurs accompagnants agrémentant parfois leur séjour de soins de bienêtre.

Cependant, si le tourisme médical peut être un levier de développement territorial, les retombées en termes de développement humain sont moins évidentes. En effet, si le développement de ce secteur entraîne une amélioration des conditions de vie des acteurs engagés dans ce secteur, cet impact reste limité car une grande partie des populations locales reste à l'écart de cette croissance économique. Il semblerait même, sous l'effet de la double dynamique de métropolisation et de privatisation de l'offre de soins, que les populations locales en subissent des préjudices du fait notamment d'un accès aux soins rendu plus difficile (Jadhav, 2014). Au sujet de l'émergence de l'Inde, Amartya Sen et Jean Drèze parlent de croissance (économique) sans développement (humain) (Drèze et Sen, 2014). Il semblerait que le tourisme médical n'échappe pas à ces forces paradoxales.

\section{Conclusion}

En raison des éléments exposés au sein de cet article, le tourisme procréatif en Inde, et plus largement vers les pays asiatiques, semblait devoir continuer à se développer de manière exponentielle. Mais on assiste, depuis le début de l'année 2015, à un mouvement important qui pourrait conduire à restreindre fortement le tourisme procréatif. En effet, plusieurs pays ont récemment décidé de réserver le recours à la gestation pour autrui à leurs seuls ressortissants et de l'interdire en conséquence aux couples de nationalité étrangère, en réaction face aux dérives suscitées par le développement d'un véritable " commerce d'enfants ». C'est le cas de la Thaïlande et de l'Etat de Tabasco au Mexique, alors que ces États étaient souvent considérés comme «l'eldorado » de la gestation pour autrui. Le Cambodge, le Népal et la Malaisie semblent suivre une voie identique de fermeture de la gestation pour autrui aux étrangers. Le recours à la gestation pour autrui deviendrait ainsi de plus en plus difficile et pourrait conduire à une réorientation des flux, voire à une réduction même si les demandes, quant à elles, augmentent. Ce retour à la dimension nationale dans certains pays s'observe également en Inde. En effet, depuis la prise de pouvoir du nationaliste Narendra Modi, des évolutions dans le domaine du tourisme procréatif commencent à apparaître. Narendra Modi a d'ailleurs déjà clairement annoncé qu'il souhaite que la gestation pour autrui soit uniquement réservée aux couples de nationalité indienne. Si 
les touristes étrangers ne peuvent plus bénéficier d'un accès à la gestation pour autrui sur le territoire indien, on assistera alors fort probablement à une nouvelle orientation des flux vers des territoires plus permissifs.

\section{BIBLIOGRAPHIE}

Anand R., Gupta S., 2008, Medical Tourism, a growth industry, Hyderabad : IFCAI University Press.

Baujard J., 2012, Intermédiaires du tourisme médical. Les réfugiés afghans de Delhi, Moussons, $\mathrm{n}^{\circ}$ 19.

Bautès N., Cadène P., Dumortier B., 2015, Le tourisme en Inde : imaginaire et réalités, in Cadène P. \& Dumortier B. (dir.), Inde : une géographie, Paris : Armand Colin, 289-303.

Bochaton A., Lefebvre B., 2008, The rebirth of the hospital : heterotopia and medical tourism in Asia, in Asia on Tour : Exploring the rise of Asian tourism, Tim Winter et al. (éds), Londres : Routledge, 97-108.

Brunet L., 2010, La gestation pour autrui : entre le marteau (de la loi) et l'enclume (des pratiques), Revue Lamy droit civil n ${ }^{\circ}$ 76, novembre 2010, 75-83.

Brunet L., 2012, Assistance médicale à la procréation et nouvelles familles : boîte de Pandore ou corne d'abondance ?, Revue de droit sanitaire et social , 828-838.

Chasles V., 2008, Femmes en Inde, L'Information Géographique, vol. 72, 57-69.

Chasles V., 2011, Se déplacer pour se faire soigner : une mobilité en expansion, généralement appelée "tourisme médical", Géoconfluences.

Chinai R., Gosawi R., 2007, Medical Visas mark growth of Indian Medical tourism. Bulletin of the World Health Organization, vol. 85, $\mathrm{n}^{\circ}$ 3, 164-165.

Drèze J., Sen A., 2014, Splendeur de l'Inde, Paris : Flammarion.

Fabre-Magnan M., 2013, La gestation pour autrui, Paris : Fayard.

Fulchiron H., 2013, La reconnaissance de la famille homosexuelle : étude d'impact, Revue Dalloz, 100.

Gany F.M., Herrera A.P., Avallone M., Changrani J., 2006, Attitudes, knowledge, and healthseeking behaviors of five immigrant minority communities in the prevention and screening of cancer: a focus group approach, Ethnicity and Health, vol. 11, ${ }^{\circ} 1,19-39$.

Gesler W. M., 1992, Therapeutic landscapes: medical issues in light of the new cultural geography, Social Science and Medicine, $\mathrm{n}^{\circ}$ 34, 735-746.

Gurtin Z.B., Inhorn M.C., 2011, Introduction: travelling for conception and the global assisted reproduction market, Reproductive biomedicine online, vol. $23, \mathrm{n}^{\circ} 5,535-537$.

Hauser J., 2014, L'externalisation de la fabrique des enfants ?, La Semaine juridique, éd. Générale (JCP G), 1770-1776. 
Heng Leng C., 2007, Medical Tourism in Malaysia : International Movement of Healthcare Consumers and the Commodification of Healthcare, Asia Research Institute, Working Paper Series $\mathrm{n}^{\circ} 83$.

Jadhav S., Yeravdekar R., Kulkarni M., 2014, Cross-border Healthcare Access in South Asian Countries: Learnings for Sustainable Healthcare Tourism in India, Procedia - Social and Behavioral Sciences, vol. 157, 109-117.

Knafou R. et Stock M. -article "tourisme", in Lévy J. et Lussault M. (dirs.), Dictionnaire de géographie et des sciences de l'espace et du social, Paris, Belin, 2003

Manaï D., 2013, Droits du patient et biomédecine, Berne : Stämpfli Editions.

Milbert I., 2015, Gujarat, in Landy F. (dir.), Dictionnaire de l'Inde contemporaine, Paris : A. Colin, 241-242.

Mouly J., 2014, La 'délocalisation procréative' : fraude à la loi ou habileté permise ?, Revue Dalloz, 2419.

Palattiyil G., Blyth E., Sidhva D., Balakrishnan G., 2010, Globalization and cross-border reproductive services: Ethical implications of surrogacy in India for social work, International Social Work September, $\mathrm{n}^{\circ}$ 53, 686-700.

Pande A., 2010, At least I am not sleeping with anyone': resisting the stigma of commercial surrogacy in India", Feminist studies, vol. 36, $\mathrm{n}^{\circ} 2,292-314$.

Pande A., 2011, Transnational commercial surrogacy in India: gifts for global sisters?, Reproductive biomedicine online, $\mathrm{n}^{\circ} 23,618-625$.

Qadeer I., Reddy S., 2010, Medical tourism in India: progress or predicament?, Economic and political weekly, vol. $45, \mathrm{n}^{\circ} 20,69-75$.

Rozée Gomez V., Unisa S., 2014, Surrogacy from a reproductive rights perspective : the case of India ", $\mathrm{n}^{\circ} 70,185-203$.

Rozée V., Tain L., 2010, Le recours aux techniques procréatives : des disparités nationales aux circuits transnationaux, in Chasles V. (ed), Santé et mondialisation, Lyon : Université Jean Moulin Lyon 3.

Schamps G., Sosson J. (dir.), 2013, La gestation pour autrui, vers un encadrement ?, Bruxelles : Bruylant.

Solomon H., 2011, Affective journeys : the emotional structuring of medical tourism in India, Anthropology \& Medicine, vol. 18, $\mathrm{n}^{\circ}$ 1, 105-118.

\section{NOTES}

1. Le Ramayana est avec le Mahabharata l'une des deux grandes épopées indiennes.

2. Apparu en Inde au 6 ème siècle avant J.C., le bouddhisme a progressivement disparu $\mathrm{du}$ pays pour se diffuser dans le reste de l'Asie. En 2011, la communauté bouddhiste représentait environ $0,7 \%$ de la population indienne.

3. A titre comparatif, les visas touristes sont valables pour une période de 6 mois maximum. 
4. La ville de Chennai, située dans l'état du Tamil Nadu, est parfois qualifiée de « capitale de la santé » puisque ce sont environ $45 \%$ des touristes médicaux qui s'y font soigner.

\section{RÉSUMÉS}

Depuis les années 1990, on assiste au développement d'un phénomène nouveau, sorte de « shopping médical international » : des personnes ou des couples se déplacent à l'étranger pour recourir à certaines prestations de soins telles que des opérations dentaires, des actes de chirurgie esthétique, des techniques d'assistance médicale à la procréation ou encore des cures de thalassothérapie. Si l'Inde n'est pas une destination touristique de premier plan, elle constitue en revanche une destination privilégiée pour le tourisme médical en général, et pour le tourisme procréatif en particulier. Les raisons qui expliquent ce phénomène sont principalement juridiques (interdiction de la pratique de la gestation pour autrui dans de nombreux pays alors qu'elle est tolérée en Inde), financières (faible coût de cette pratique en Inde) et techniques (développement d'une offre de qualité en Inde, bénéficiant d'une labellisation internationale). L'article se propose de dresser un bilan des flux générés par le recours à la gestation pour autrui en Inde et d'analyser tant les causes à l'origine de ces flux que les conséquences en termes de développement touristique des territoires.

Since the 1990s, there has been the development of a new phenomenon, a kind of "international medical shopping ": individuals, people or couples traveling abroad to have access to certain health services such as dental operations, acts of cosmetic surgery, thalassotherapy cures or techniques of medically assisted procreation. India is has become one of the worldwide destinations for medical tourism in general and for reproductive tourism in particular.

Medical tourism emerged as a growing sector in India in the 1990s as a result of liberalization and globalization of the Indian economy. The country is one of three main destinations for medical tourists in Asia, together with Thailand and Malaysia. The pull factors are the low cost of care combined with high quality hospital services and the high level of competence of doctors. This sector is mainly supported by large private hospital groups. The international patients come mainly from Asia, especially Bangladesh and Sri Lanka but also from Malaysia and Pakistan. There are also patients from developed countries (USA, UK) including among these, those with origins in the indian diaspora. The most sought care belong to various fields of surgery (cardiac surgery, bone marrow transplants, hip replacements, eye surgery and so forth) and care for wellness inspired by traditional Ayurvedic medicine.

In recent years, India has become an increasingly popular destination in the field of assisted reproduction, and particularly in that of surrogacy. This element within a broader medical tourism differs markedly in the logic that contributes to the choice of India as the place of care. Indeed, the main reason is that surrogacy is legal in India in comparison with the ban on the practice in many countries. The lack of comprehensive and reliable data does not accurately assess the importance of this practice in India. However, the few studies in this area agree on the speed of its growth. India's provision of reproductive services attracts reproductive medical tourists from the so-called Western countries along with many from Sri Lanka, Pakistan, Bangladesh, Thailand and even Singapore. In total, some sources estimate that nearly 12,000 foreigners come every year in India to resort to surrogacy reproductive services. 
Nationally, while these clinics are present in the whole of India, it is Gujarat State which enjoys the greatest visibility in this area and which has been dubbed the "world's cradle".. This contrasts the patterns by state of general tourism or even wider medical tourism, in which Gujurat does not feature as a lead state of choice. In 2014 , the state received only $2.4 \%$ of domestic tourists (about 31 million people) and 1.04\% of foreign tourists (about 236,000 people). And as indicated above, Gujarat is not one of the preferred destinations for medical tourists, the latter preferring the cities of Chennai, Delhi, Mumbai or even Bangalore. So, today, Gujarat is a State specialized in a very specific field of medical tourism, that is surrogacy.

So, through the example of India, this article aims first to show how the reproductive tourism geography obeys a different logic from that of medical tourism. From the example of surrogacy, it will then propose an inventory of this practice in India and an assessment of the impact on the areas concerned, with particular reference to infrastructure development of health and tourist amenities.

INDEX

Mots-clés : gestation, tourisme de santé, reproduction

Index géographique : Inde

\section{AUTEURS}

VIRGINIE CHASLES

MCU en géographie, Université Jean Moulin Lyon 3, EA HESPER

MARION GIRER

MCU HDR en droit privé, Université Jean Moulin Lyon 3, IFROSS 